\title{
Regression model for computing leaf area and assessment of total leaf area variation with frond ages in oil palm
}

\begin{abstract}
In this study a regression models for accurate estimation of leaf area from simple measured leaves length and middle width were described as well as assessment of total leaf area variation with frond ages. Results shows that total leaf area of the frond are decreased with increase frond ages and upper frond represents more leaf area than lower frond at same palm. In this study two models (linear and log-linear) were proposed for accurate estimation of leaf area. (a) $\operatorname{Lac}=0.80 \times$ (L.W) And (b) $\log \operatorname{Lac}=0.957 \times \log (\mathrm{L} . \mathrm{W})$, where Lac, L and W represents the actual leaf area, leaf length and leaf width respectively. Statistical analysis indicates a high degree of association $(\mathrm{R} 2=0.99)$ and the low standard errors of estimation were 0.7477. The standard error of estimate of coefficient was 0.0032 (model áâ). Logarithmic transform of data were also well fitted both linear and non-linear regression. However, it is considered only linear model for simplicity. These Logarithmic transform of data also indicate a high degree of association $(\mathrm{R} 2=0.99)$ and the low standard error of estimation were 0.02 . The standard error of estimate of coefficient was (0.0005). This model was validated using other experimental results, which showed a good agreement between measured and estimated leaf area.
\end{abstract}

Keyword: Regression model; Leaf area variation; Oil palm frond 